\title{
Incorporating Machine Learning into Vibration Detection for Wind Turbines
}

\author{
J. Vives \\ Institute of Automatic and Industrial Informatics, Universitat Politècnica de València, Valencia 46022, Spain \\ Correspondence should be addressed to J. Vives; javifus@posgrado.upv.es
}

Received 20 October 2021; Revised 30 January 2022; Accepted 3 February 2022; Published 16 February 2022

Academic Editor: Nicolò Vaiana

Copyright (c) 2022 J. Vives. This is an open access article distributed under the Creative Commons Attribution License, which permits unrestricted use, distribution, and reproduction in any medium, provided the original work is properly cited.

\begin{abstract}
With machine learning techniques, wind turbine components can be detected and diagnosed in advance, so degeneration can be prevented. Automatic and autonomous learning is used to predict, detect, and diagnose electrical and mechanical failures in wind turbines. Based on the implementation of machine learning algorithms adapted to the different components and faults of wind turbines, this study evaluates different methodologies for monitoring, supervision, and fault diagnosis.
\end{abstract}

\section{Introduction}

Due to global warming and the significant increase in energy demand and consumption, the transition to obtaining electricity from renewable sources is accelerating. Researchers and engineers have developed new specific techniques for maintaining wind power plants as wind power production has grown by around $25 \%$ in recent years. The reliability, safety, and profitability of wind turbines can be improved with efficient methods of advanced monitoring and fault diagnosis.

In maintenance of wind turbines [1], the main fault methodologies are spectral analysis and fault trees, but with all the additional technological advances that this entails, connectivity, smart, and data generation, we are seeing a transformation in maintenance towards artificial intelligence (AI) and machine learning.

Increasingly, data are available to the industry at this point, which affects critical decisions in crucial areas such as scheduling [2], maintenance management [3], and quality improvement [4].

Recent developments in hardware, cloud-based solutions, and a new generation of algorithms have amplified the impact of machine learning in these areas [5]. There are many components in a wind turbine that work together, and vibration is one of the primary causes of the system's failure.
Vibrations usually indicate mechanical or electrically related faults, as well as gear or bearing defects.

It is the rolling elements of bearings that are prone to greater wear, since their surface position changes continuously with respect to their load, causing a behaviour corresponding to speed of rotation.

Additionally, geometric imperfections or roughness contribute to vibrations. Normal operating conditions do cause not only vibrations but also faults like exterior and interior raceways, component failures, cage failures, imbalance, and misalignments.

Several studies have been conducted on vibrations in structures as well as in rotating machines. The majority of research, studies, and results in this field to date are based upon traditional methods, that is, frequency spectral analysis, as a means of detecting bearing failure due to mechanical vibrations. From the studies performed on wind turbines [6], it appears that conventional diagnostic techniques traditionally have been used to detect faults in the generators and in their structures. The studies and results that are provided by AI [7], in this case machine learning, show that this type of methodology has worked and continues to work perfectly, but it has limitations and drawbacks.

The industry is implementing this new approach. In case of an anomaly in the mechanical components of a machine, it is possible to anticipate, detect, and classify it autonomously in line with this series of maintenance 
methodologies. Regarding [8], machine learning can lead to a reduction of response time and an almost complete elimination of error possibilities.

Additionally, according to [9], data management and analysis facilitate feedback learning and enable offshore implementations to be flexible. The AI methodology protects you from any type of failure that you desire to monitor by analysing and preventing it. In order for these methods to be implemented on a real system without causing costly errors, they must be adequately validated. It is therefore convenient to validate fault diagnosis techniques by using prototypes or test benches. In order to develop new techniques, conduct studies, etc., these prototypes are used in order to understand how these systems work.

One or more wind turbines going down can result in a considerable loss of money for two reasons: first, for the cost of replacement, and second, for the energy that cannot be produced while the wind turbine is stopped, which may happen in peak energy production seasons. In wind farms, where repair and maintenance costs are very high, especially in offshore locations, fault detection and diagnosing techniques are a necessity, since it is essential to stop the machine as soon as possible. Additionally, to lower costs associated with downtime and defective products, it is becoming increasingly important to manage maintenance activities efficiently.

By comparison to existing systems, we have a prototype that can detect, supervise, and anticipate failures through the application of algorithms designed to anticipate and prevent potential problems using a machine learning methodology. In this paper, we propose a feature-learning method for detecting different bearing failures autonomously using vibration analysis.

In this article, we present the application of an intelligent algorithm applied to a prototype of a wind turbine for the supervision and diagnosis of faults. A review of the literature is given in the next section. Subsequently, the data capture procedure and data set are discussed. The results of the classifiers used are then evaluated and compared. Finally, some relevant conclusions of the study are presented.

\section{Working Methodology}

There are different methodologies for diagnosing and monitoring failures in wind turbines due to vibrations in bearings, obtaining multiple characteristics of a bearing as a result; therefore, the characteristics extracted by the general methods of a bearing do not necessarily correlate with the extracted fault characteristics of another bearing, following the same or different methodology. This study illustrates how the use of machine learning techniques can allow for greater accuracy and prediction of possible breakages or anomalies by using the characteristics extracted from vibration measurements.

2.1. Machine Learning. For the detection of faults in wind turbines, machine learning focuses primarily on two tasks, the first being the detection of anomalies and the second being the classification of faults. With this technique, it is possible to detect failures promptly or anticipate them, allowing for corrective measures to be taken in a very short time, significantly improving the reliability and security of the system (Table 1).

In the world of machine learning, there are two types of methods: supervised and unsupervised. The most common machine learning use case, by far, is supervised machine learning [10]. With supervised learning, the output of your algorithm is already known, while with unsupervised learning, it is not. To get in, on the way out, you only need to figure out the process. In most cases, algorithms are "taught" from training data sets. Unsupervised learning, on the other hand, is a more complex process, because it relies only on input data and binary logic that all computer systems use.

To apply any type of learning, the data must first be classified [11].

Different classification algorithms can be applied to this problem, where they take the functionality of an object and identify it by a limited number of categories or classes from input information received from that object.

As a result, a classifier works in two phases:

(i) To achieve optimal performance, it must be trained, which means receiving a large amount of sample data and its correct classification, so as to adjust its parameters

(ii) When the algorithm has already been trained, it provides output based on the input data it receives

2.2. Support Vector Machine. An SVM is a machine learning algorithm based on statistical learning theory. This method works well for classification and regression, such as in fault diagnosis, when we use small samples. It is shown that a linear classifier can separate two simple classes. These two types of samples are represented by triangles and squares in Figure 1. Two classes can be separated by a hyperplane $H$. In these two classes, the planes $H_{1}$ and $H_{2}$ (shown in dashed lines) are parallel to $H$ and pass through samples that are closest to $H$. Margins are calculated by taking the distance between $H_{1}$ and $H_{2}$. In the SVM, linear boundaries are placed between two distinct classes $H_{1}$ and $H_{2}$. The margin is maximized so the generalization error is the smallest. Support vectors are often used to measure margins, and they include the closest points to the margin.

A quadratic function is minimized under linear inequality constraints by reducing it to convex optimization [12]. Assume we have a training set of samples $\left[\left(x_{i},\left(y_{i}\right)\right]\right.$, where $i=1$ to $N$ and $N$ represents the total number of samples. In order to find the separation plane with the least generalization error out of each linear separation plane, we need to determine how to divide the input samples into two classes. It is possible to divide the samples into two classes: triangular and square. A triangle class has a $y_{i}=-1$ label. A square class has a $y_{i}=+1$ label. For nonseparable data, slack variables are not considered (nor $P 0$ ). Using the following optimization problem, you can obtain the hyperplane for $f$ $(x)=0$ from the given data: 
TABLE 1: Advantages and disadvantages of KNN and SVM algorithms.

\begin{tabular}{|c|c|c|}
\hline Algorithm & Advantages & Limitations \\
\hline KNN & $\begin{array}{c}\text { Easy to implement } \\
\text { Good overfitting } \\
\text { Robustness to noisy data } \\
\text { Can be used for both classification and regression }\end{array}$ & $\begin{array}{c}\text { Great computation } \\
\text { Slow sorting speed } \\
\text { Needs a lot of storage space } \\
\text { The selection of } k \text { influences the classification }\end{array}$ \\
\hline SVM & $\begin{array}{c}\text { High sorting accuracy } \\
\text { Fast sorting speed } \\
\text { Can handle high-dimensional features } \\
\text { Robust to overfitting and noise }\end{array}$ & $\begin{array}{l}\text { Low efficiency for big data } \\
\text { Binary classifier } \\
\text { No physical meaning }\end{array}$ \\
\hline
\end{tabular}

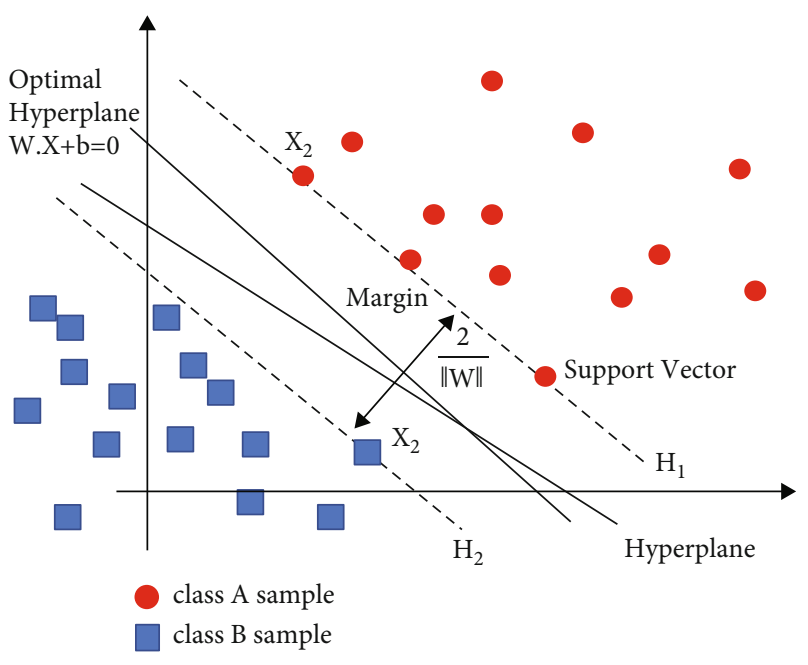

FIgURE 1: Optimal hyperplane for binary classification by SVM.

$$
\begin{gathered}
\text { Minimize } \frac{1}{2}\|w\|^{2}+C \sum_{i=i}^{N} \xi_{i} \\
\text { Subject to }\left\{\begin{array}{l}
y_{i}\left(w^{T} x_{i}+b\right) \geq 1-\xi_{i}, \\
\xi_{i} \geq 0, \quad i=1,2, \cdots, N,
\end{array}\right.
\end{gathered}
$$

where $C$ is a constant representing the error penalty. Introducing Lagrange multipliers to the optimization problem above leads to the following result:

$$
\begin{gathered}
\text { Minimize } W(\lambda)=\sum_{i=i}^{N} \lambda_{i}-\frac{1}{2} \sum_{i j=i}^{N} y_{i} y_{j} \lambda_{i} \lambda_{j}\left(x_{i} x_{j}\right) \\
\text { Subject to }\left\{\begin{array}{l}
0 \leq \lambda_{I} \leq C, \\
\sum_{i=i}^{N} \lambda_{i} y_{i}=0, \quad i=1,2, \cdots, N .
\end{array}\right.
\end{gathered}
$$

Using the sequential minimum optimization (SMO) algorithm, the dual problem that results from SVM derivation can be efficiently solved. SMO breaks down the general QP problem into QP subproblems.

2.3. K-Nearest Neighbor (KNN). Learning algorithms based on these principles can help users determine how several instances within a data set experience similar characteristics [13]. Learning occurs at the same time that test data is tested,

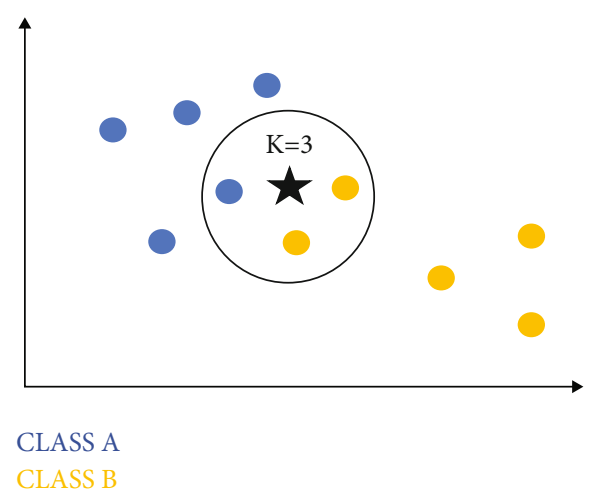

Figure 2: KNN diagram with different samples.

so that rather than creating a model from learning with training data, the model is created automatically. Lazy learning is another name for this algorithm type.

Its operation is very simple, for a given training group of classified instances $T=\left[\left(x_{1}, y_{1}\right),\left(x_{2}, y_{2}\right), \cdots,\left(x_{N}, y_{N}\right)\right]$, where $x_{i}$ is the vector of characteristics of the unlabeled instance and $y_{i}$ is the label $y_{1}=c_{1}, c_{2}, \cdots, c_{K}, i=1,2, \cdots, N$. Using a given distance metric, the KNN algorithm finds the $k$ closest instances to a training sample $(x, y) . N_{k}$ represents the area where these $k$ instances are located. As a result, it is possible to calculate the test sample label $x$ from the decision rules:

$y=\arg \max _{c j} \sum_{x_{i} \varepsilon N_{k}(x)} I\left(y_{i}=c_{j}\right), \quad i=1,2, \cdots, N, j=1,2, \cdots, K$,

where $I$ is the indicator function.

According to Figure 2, by analysing what an unclassified instance's closest neighbors are, we can extract its tag.

Three basic concepts make up the KNN algorithm: how many instances were measured, the classification decision rule, and how many measured instances there were.

\section{System Description}

In this section is described the industrial environment within which the system will operate and list each component it comprises. It also describes the distribution of the sensors. The document also discusses the features of a data acquisition card, which measures signals and their connections. 


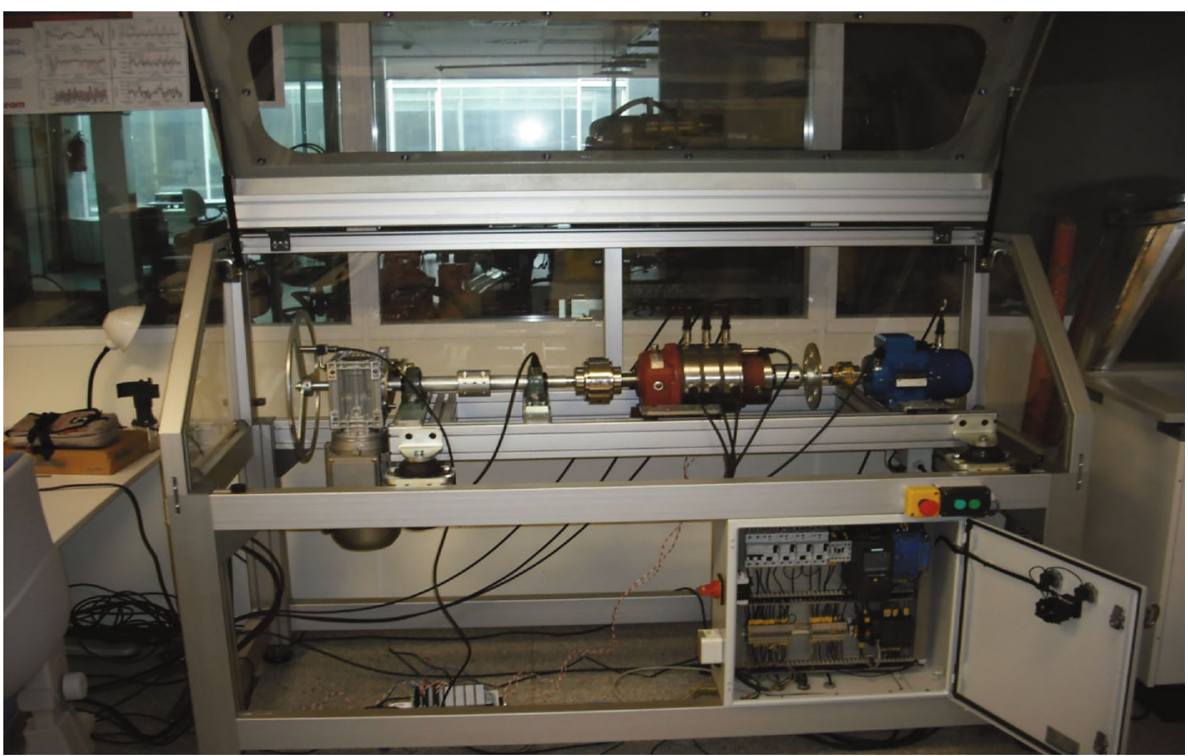

Figure 3: Component distribution in the prototype.

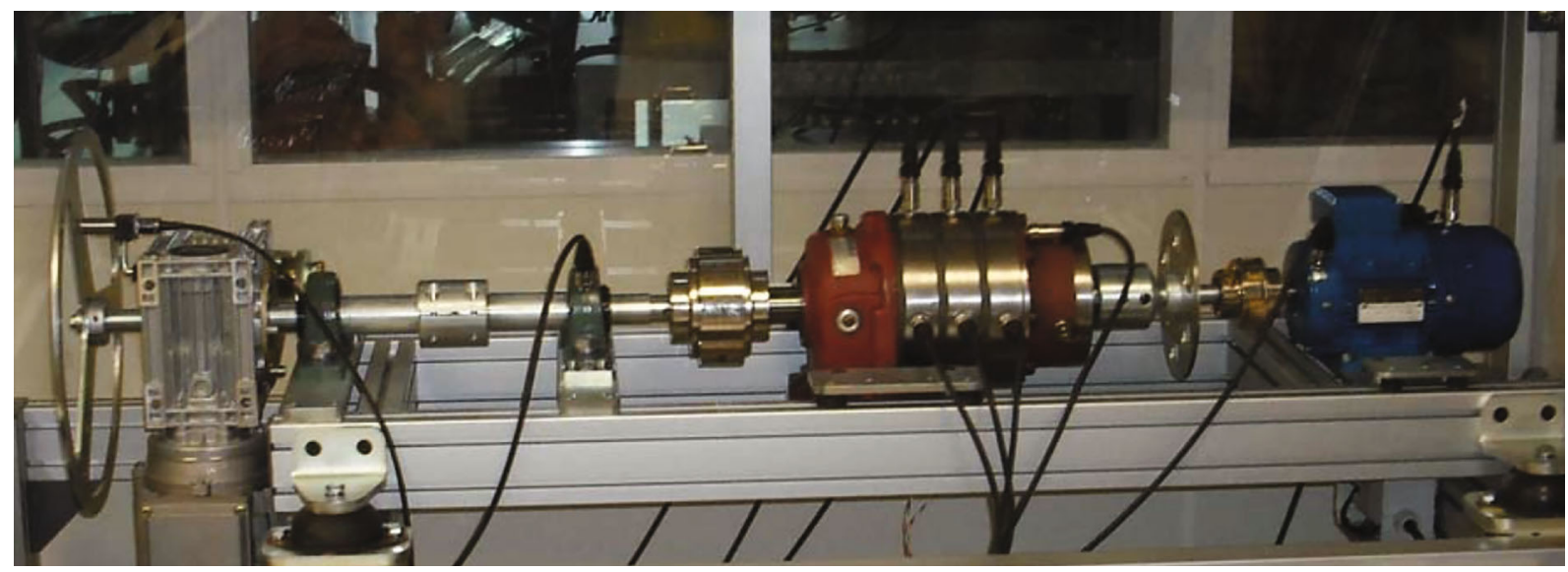

Figure 4: Position of the accelerometers in all the systems.

3.1. Prototype. As shown in Figure 3, small wind turbine prototypes are very useful and provide valuable data for diagnosing failures in its components. For instance, it provides information for watching for deterioration and wear in its parts and also determining the effects that it has [14]. Designed towards this end, the system allows for parts to be exchanged without any difficulty, without the need to wait for deterioration to take place, which enables testing diagnostic techniques without waiting for deterioration.

3.2. Distribution on the Prototype. We measure the vibration of the generator, gearbox, and bearings. In the case of a generator, it is recommended that the vibration sensors be located in the input bearing of the machine, in order to study the vibrations at the fast shaft coupling. Considering the multiplier, according to the techniques used for state monitoring and the design of the machine, each stage should have its own sensor, which will allow the analysis of signal propagation between each device, as well as how each part failure affects vibrations within the machine.
Another interesting feature of the prototype is its slow axis bearing, which is ideal for measuring. Furthermore, this element can be changed for another exactly the same with deteriorated bearings and thus be studied under the conditions of use in order to study the characteristics of the signal in normal operation and the degeneration of the component itself.

The following distribution of 10 accelerometers (Figure 4) was thus decided, based on the above considerations.

3.3. Data Acquisition Card. Vibrations are measured by accelerometers. They come with 2-pin MIL-C-5015 NI connectors ideal for general purpose accelerometers. The acquisition card PCI-4472B [15] was used, optimized for vibration measurements, which provides high-precision frequency-domain measurements through its eight-channel dynamic signal acquisition (DSA). For accelerometers and microphones, IEPE-based signal conditioning is integrated into input channels. Input signals from DC to $45 \mathrm{kHz}$ are simultaneously digitally digitized across the eight input 


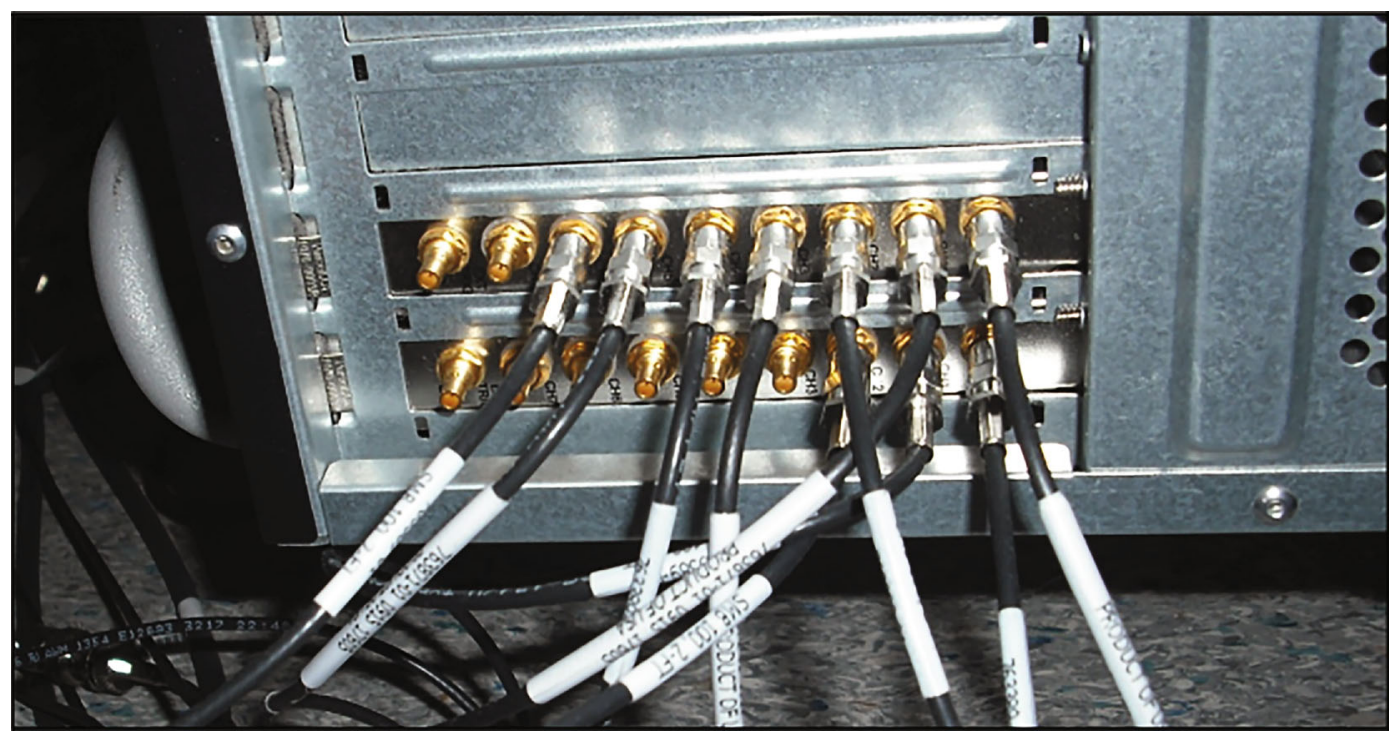

Figure 5: Connection of accelerometers to the data acquisition system.

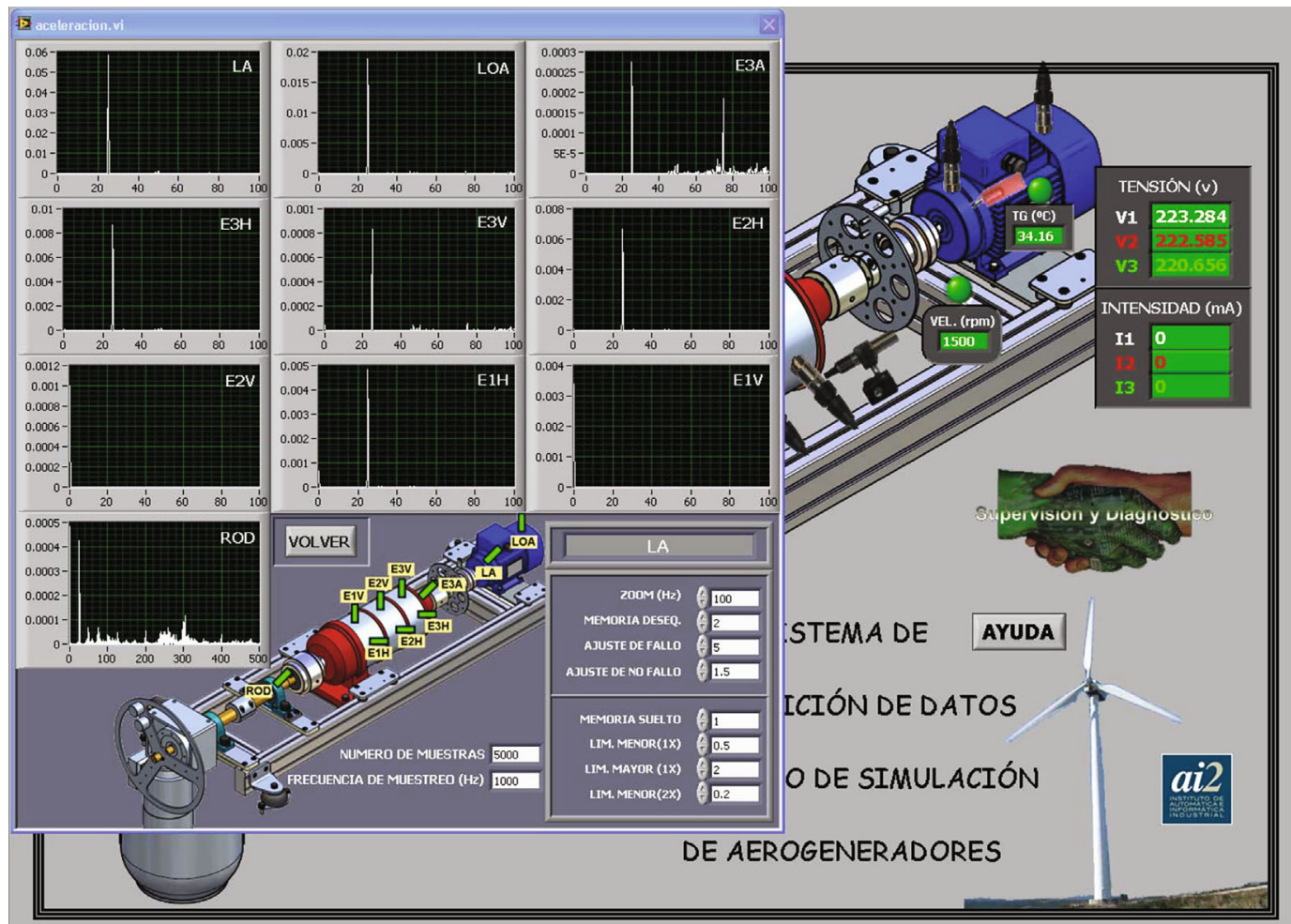

FIGURE 6: System vibration detection.

channels. For very low-frequency AC vibration measurements, the PCI-4472B features a cut-off frequency of just $0.5 \mathrm{~Hz}$. Software available from NI is designed for sound and vibration measurement and analysis. It provides audio measurements, octave analysis, frequency analysis, transition analysis, and order tracking. As explored previously, the acquisition system relies on two PCI-4472B modules because the prototype has a total of 8 inputs and ten accelerometers.
3.4. Connection with the Data Acquisition System. National Instruments recently developed a PCI-type card specifically designed for measuring vibration by means of accelerometers. This card can be mounted similarly to any other PCI device. This card contains 8 inputs for accelerometers and is called the NI PCI-4472B. The connections are shown in Figure 5.

In Figure 5, you can see that 3 accelerometers are mounted on the lower card, while the remaining 7 are 


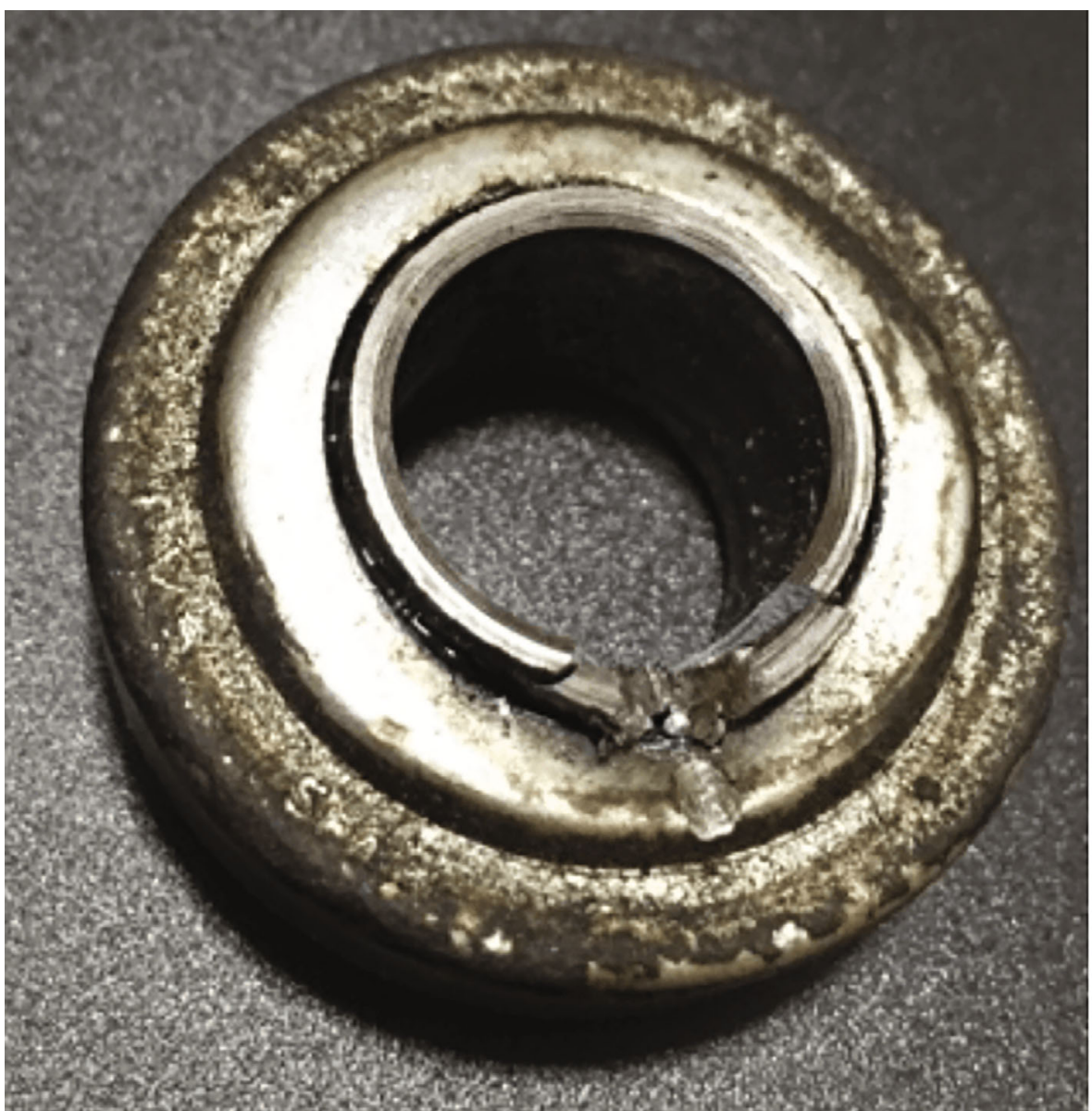

Figure 7: Broken bearing.

mounted on the upper card. There are three accelerometers in the multiplier, one in the bearing and two in the first stage (E1H and E1V), for which the acquisition must be performed much more slowly than for the rest.

\section{Results}

We make a comparison between the traditional approaches and those of artificial intelligence in this section. Traditionally, vibratory motions are measured using spectral analysis. Figure 6 illustrates the results of applying a classical spectral analysis. The simulation was performed successfully. In Figure 6, you can see the output of the 10 accelerometers around the wind turbine prototype. This prototype can rotate at 5 different speeds, between zero and $1500 \mathrm{rpm}$. In this situation, a moderate speed of $300 \mathrm{rpm}$ has been used. An average of 5000 samples was obtained for each sensor analysed, and a sampling frequency of $1 \mathrm{kHz}$ was utilized for the graphic representation.

Technological advancement is transforming classical vibration analysis methodologies into more automated and precise methodologies. Wind turbine failures can be tracked, prevented, and diagnosed with automatic learning systems. A correct automatic prediction relies largely on training the algorithm so that it has feedback and is able to work autonomously through data analysis and classification after the algorithm has received feedback. This section outlines the basic aspects of teaching and training the algorithm to ensure it has reliable results. We have trained this algorithm about eight times in total, and we consider that sufficient feedback for prediction. During training, the 2 states of analysis were simulated, breakage (Figure 7) and an imbalance.

A final comparison is conducted between the two states for each of the two classification methods, first $K$-nearest neighbor (KNN) (Figure 8) and second Support Vector Machines (SVM) (Figure 9). We divided it into 4 phases to obtain it. For the first step, the data were obtained via the acquisition card PCI-4472B, for subsequent processing and filtering. For the analysis to be stable, it is essential to transform the signal into something nonrandom. When applying machine learning algorithms to these types of signals, appropriate conditioning and efficient processing are crucial to extracting patterns from them. For this method to function correctly, another key aspect is that the signal's time variation makes it difficult to process and to learn from. For the algorithm to function correctly, this first stage of signal conditioning and filtering is crucial. In this first point, the signal processing should be such that the algorithm reads invariant characteristics in time. 

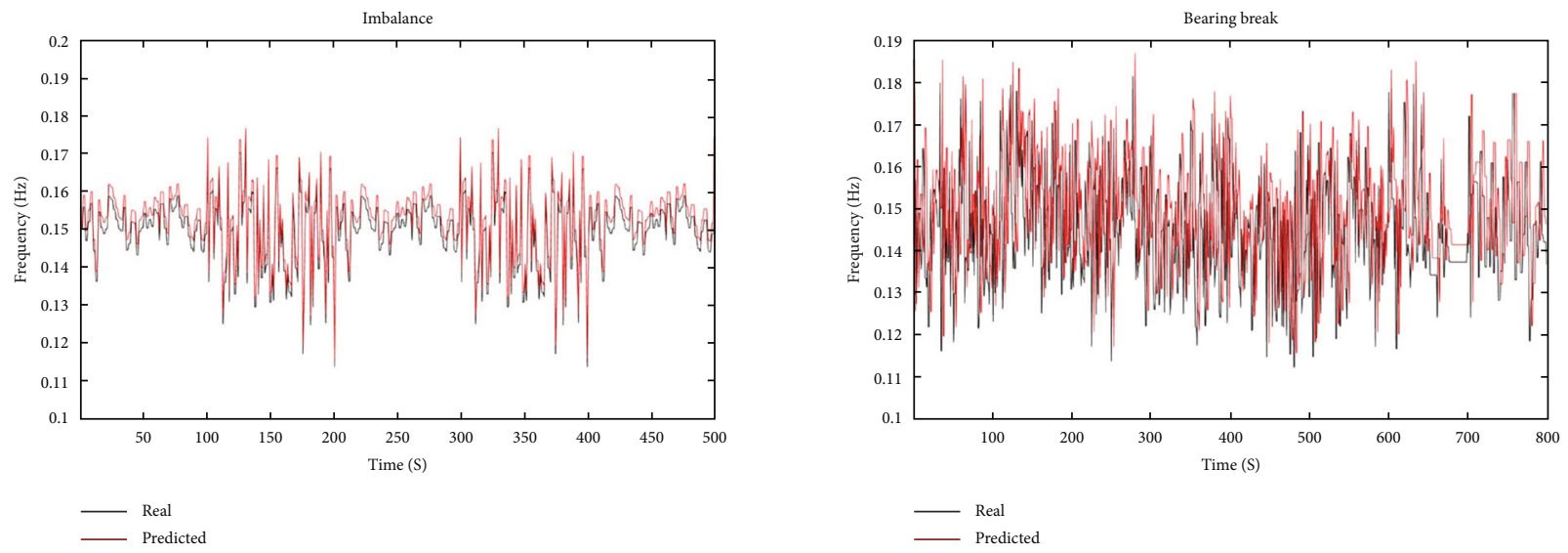

FIGURE 8: Real output vs. predicted output $K$-nearest neighbor algorithm.


FIgURE 9: Real output vs. predicted output Support Vector Machine (SVM) algorithm.

The faults and conditions introduced are

(i) imbalance

(ii) bearing break failure

To determine the failure threshold or stipulated condition for each failure or condition, extraction of the characteristics must be completed. Let us examine each of these phases in more detail. In order to calculate the arithmetic mean, each of the acquired samples has been summed together (for each of the stated failure conditions) and divided by the total number of samples studied. The data set is then reduced in dimension by conducting a principal component analysis. This method reduces the number of variables so as to represent the old ones as accurately as possible with the smallest number of new variables. Our state is then understood, as well as what it is doing, helping us to make future decisions. The standard deviation of each of the stipulated failure conditions is obtained by using the results of the two previous phases. The data presented in it demonstrate variation or dispersion. The standard deviation of each state has been relatively low, which implies the majority of points are close to the mean, meaning it should work correctly.
Both classifiers use these three stages. In the following phase, both methodologies of the two algorithms come into play in order to classify the data. This occurs after data have been correctly conditioned.

Following this entire process, the algorithm is given several training rounds, thus enabling self-operation in the future. The algorithm is ready to work after several training sessions; it only requires new data. Special analysis and classification of these new data help predict the process being controlled.

Here is a breakdown of each state. First is the failure due to an imbalance, both classifiers follow more or less the same pattern, but in this case, in the SVM algorithm, the results are a little out of date with respect to their feedback, due to the limits used for this case. Finally, the failure is found due to the breakage of the bearing race; both algorithms no longer behave in the same way. The data analysed for the KNN are more grouped than the SVM; this is due to the different ways of classifying and analysing the data, because they do not follow a certain pattern.

In addition, both algorithms have excellent accuracy and similarity to the real and predicted output for either of the proposed failure conditions. It is observed that the results 
of the two classifiers are similar and do not differ much from one another.

For example, let us focus on the imbalance variable. In $96 \%$ of cases, this variable is correctly classified, while in $4 \%$ cases, it is incorrectly classified. Specifically, over $90 \%$ of true positives were detected, with less than $10 \%$ false positives. Support Vector Machines (SVM) yielded a 95\% success rate, while KNN reached $94 \%$. As a result, both the KNN and SVM learning algorithms are thought to have a lot of similarities with our wind turbine prototype, allowing us to accurately predict when the turbine will fail.

\section{Conclusions}

Machine learning is improving the accessibility and reliability of wind turbine fault detection, monitoring, and diagnosis. Data acquisition and classification are vital to the success and proper functioning of artificial intelligence. As a result of the analysis of vibrations and the use of AI techniques, this document examines several approaches to diagnosis and prevention of failures in wind turbine bearings. From a theoretical and a practical perspective, we have summarized the fault diagnosis for bearings based on the KNN and SVM models. Both of these models feature high processing speeds, robustness, and very high precision, which are advantages especially for this kind of study. Traditional methods, such as spectral analysis, are being displaced by their advantages as well as their ease of classification and prediction. This methodology has been shown to be highly successful in predicting stipulated failure conditions, allowing it to be applied to other mechanical components of the prototype, with the aim of preventing or anticipating possible wind turbine breakdowns. A prototype implementation simulates typical wind turbine faults by replacing worn-out parts with other defective or worn ones. New methods of fault diagnosis and supervision can be evaluated, developed, and validated with these study possibilities. It is therefore very helpful to use prototypes of wind turbines in order to test designed diagnostic algorithms, in the phase before their installation in high-power wind turbines, to reduce costs and time, and to make necessary verifications, adjustments, and corrections, thus increasing their accuracy and reliability.

\section{Data Availability}

No data were used to support this study.

\section{Conflicts of Interest}

The author declares that they have no conflicts of interest.

\section{References}

[1] J. Y. Hsu, Y. F. Wang, K. C. Lin, M. Y. Chen, and J. H. Y. Hsu, "Wind turbine fault diagnosis and predictive maintenance through statistical process control and machine learning," Ieee Access, vol. 8, pp. 23427-23439, 2020.

[2] L. Wang, Z. Liu, Q. Miao, and X. Zhang, "Time-frequency analysis based on ensemble local mean decomposition and fast kurtogram for rotating machinery fault diagnosis," Mechanical Systems and Signal Processing, vol. 103, pp. 60-75, 2018.

[3] M. Abdel-Nasser, K. Mahmoud, and H. Kashef, "A novel smart grid state estimation method based on neural networks," IJIMAI, vol. 5, no. 1, pp. 92-100, 2018.

[4] L. D. S. Leão, A. A. Cavalini Jr., T. S. Morais, G. P. D. Melo, and V. Steffen Jr., "Fault detection in rotating machinery by using the modal state observer approach," Journal of Sound and Vibration, vol. 458, pp. 123-142, 2019.

[5] O. Janssens, V. Slavkovikj, B. Vervisch et al., "Convolutional neural network based fault detection for rotating machinery," Journal of Sound and Vibration, vol. 377, pp. 331-345, 2016.

[6] R. Zhao, R. Yan, Z. Chen, K. Mao, P. Wang, and R. X. Gao, "Deep learning and its applications to machine health monitoring," Mechanical Systems and Signal Processing, vol. 115, pp. 213-237, 2019.

[7] J. Franko, S. Du, S. Kallweit, E. Duelberg, and H. Engemann, "Design of a multi-robot system for wind turbine maintenance," Energies, vol. 13, no. 10, p. 2552, 2020.

[8] A. H. Elkasem, S. Kamel, A. Rashad, and F. J. Melguizo, "Optimal performance of doubly fed induction generator wind farm using multi-objective genetic algorithm," IJIMAI, vol. 5, no. 5, pp. 48-53, 2019.

[9] M. B. Kane, "Machine learning control for floating offshore wind turbine individual blade pitch control," in 2020 American Control Conference (ACC), pp. 237-241, Denver, CO, USA, 2020.

[10] J. Adams, Y. Qiu, Y. Xu, and J. C. Schnable, "Plant segmentation by supervised machine learning methods," The Plant Phenome Journal, vol. 3, no. 1, article e20001, 2020.

[11] A. U. Osarogiagbon, F. Khan, R. Venkatesan, and P. Gillard, "Review and analysis of supervised machine learning algorithms for hazardous events in drilling operations," Process Safety and Environmental Protection, vol. 147, pp. 367-384, 2021.

[12] M. A. Myszczynska, P. N. Ojamies, A. Lacoste et al., "Applications of machine learning to diagnosis and treatment of neurodegenerative diseases," Nature Reviews Neurology, vol. 16, no. 8 , pp. $440-456,2020$.

[13] C. Lytridis, A. Lekova, C. Bazinas, M. Manios, and V. G. Kaburlasos, "WINkNN: windowed intervals' number kNN classifier for efficient time-series applications," Mathematics, vol. 8, no. 3, p. 413, 2020.

[14] Z. Dzulfikri, N. Nuryanti, and Y. Erdani, "Design and implementation of artificial neural networks to predict wind directions on controlling yaw of wind turbine prototype," Journal of Robotics and Control (JRC), vol. 1, no. 1, pp. 20-26, 2020.

[15] Y. Yang, S. Ma, J. Wu, J. Bowen, W. Li, and X. Luo, "Frequency feature learning from vibration information of GIS for mechanical fault detection," Sensors, vol. 19, no. 8, 2019. 